\title{
Extrinsic Current and Flash Sensitivity in Turtle Cones
}

\author{
DANIEL G. GREEN,* DAVID M. SCHNEEWEIS,*† MARILYN J. GLOVER* \\ Received 14 December 1992; in revised form 24 May 1993
}

\begin{abstract}
The effects of hyperpolarizing current and background light on intracellular responses of red cones in turtle were compared. Even though a background light always reduced response amplitude, hyperpolarizing current did so in only $25 \%$ of the cells studied. When hyperpolarizing current reduced response amplitude it also produced changes in response kinetics and the intensity-response relationships, but these changes differed from those produced by background light. Considerably greater hyperpolarization was required with current than with light to produce equivalent reductions in amplitude. The results suggest that current reduces amplitude by activating a membrane conductance, while background light acts through a different mechanism.
\end{abstract}

Adaptation Cone Current Retina Turtle

\section{INTRODUCTION}

There is extensive electrical coupling between turtle cones (Baylor, Fuortes \& O'Bryan, 1971; Baylor \& Hodgkin, 1973; Owen \& Copenhagen, 1977; Detwiler \& Hodgkin, 1979). Consequently the voltage response to a large spot of light centered on an impaled cone is considerably greater than that to a small spot of equal intensity. Most of the increased response to the larger spot is due to the coupling between cones, though some small part reflects the additional scattered light that falls upon the impaled cone.

When Baylor and Hodgkin (1974) studied thc adapting effects of disks of various sizes on a small test flash they obtained a rather unexpected finding which has since been confirmed by others (Copenhagen \& Green, 1985, 1987; Itzhaki \& Perlman, 1987). They found that when a steady background spot reduced sensitivity to a $14 \mu \mathrm{m}$ test flash by the factor $1 / \mathrm{e}$, the associated steady hyperpolarization was always about $3 \mathrm{mV}$ independent of the size of the adapting spot. Since the cones are coupled, the amount of light falling on the outer segment of the impaled receptor is different for backgrounds of different size that produce equal steady hyperpolarizations. This is because the summated effect of a dim, large background that falls on many coupled cones is equal to that of a smaller and brighter background that excites a few cones. The implication is that the effects of the large adapting background cannot be accounted for solely by the photons absorbed in the outer segment of the impaled cell and that the photons

\footnotetext{
*Department of Ophthalmology, University of Michigan, 1103 E. Huron Street, Ann Arbor, MI 48104-1687 U.S.A.

†Present address: Department of Ophthalmology, University of California, San Francisco, CA 94143-0730, U.S.A.
}

that fall on neighbors are in some way contributing to desensitization. We will refer to the adaptation mediated at a distance as remote adaptation.

The close association between the membrane potential and the flash sensitivity of an impaled cone raises the possibility that membrane potential can somehow control flash sensitivity. In this case remote adaptation would be mediated by the hyperpolarization produced by the excitation of neighboring coupled cones. The experiments described here test this idea. If the membrane potential itself causes remote adaptation then hyperpolarizing the photoreceptor with extrinsic current should mimic the adapting effect of light. We report here that while hyperpolarizing current can reduce the magnitude of the light response, this effect is not capable of accounting for the remote desensitizing effects of light.

\section{METHODS}

Intracellular potentials were recorded with microelectrodes (borosilicate glass, Sutter \# BF100-50-15) from red-sensitive cones of the snapping turtle, Chelydra serpentina, using a dark-adapted eyecup preparation. The eyecup preparation, with details of the optical and recording systems, and the procedures used for aligning the microelectrode with the stimulus beam have been described in detail elsewhere in Copenhagen and Green $(1985,1987)$. A Colburn and Schwartz (1972) amplifier was used to pass hyperpolarizing current through the recording electrode. The current was turned on manually.

Responses were digitized (sampling rate $500 \mathrm{~Hz}$ ) for analysis by computer. Response amplitude and time to peak were obtained automatically from the parameters of a parabola fit in a weighted least-squares sense to a $100 \mathrm{msec}$ window near the nominal peak of the response. The isolated retina preparation is new and the 
iments went as follows. The eyes from well-dark-adapted red-eared turtles were removed and placed in cold Ringers solution $\left(4^{\circ} \mathrm{C}\right)$ for about $2 \mathrm{hr}$. After hemisecting the eye, a pie-shaped piece was cut from the dorsal eyecup and placed retina-down on a piece of Millipore filter paper. Three minutes later the sclera and RPE could be peeled away, leaving the retina on the filter paper. The isolated retina experiments were done on the stage of a microscope (Nikon Optiphot) fitted with an IR camera so that photoreceptors could be impaled with microelectrodes under visual control. Ordinary microelectrodes (same as above) or thick-walled theta microelectrodes (boroscilicate glass, WPI \# TST150-6) were advanced into the retina and cones were impaled singly, with thick-walled theta electrodes, or in pairs with two ordinary microelectrodes. Theta electrodes had resistances of $200-500 \mathrm{M} \Omega$ in each barrel, and a coupling resistance between barrels of $<5 \mathrm{M} \Omega$. The input resistance measured in cones $(50-150 \mathrm{M} \Omega)$ was always greater than the coupling resistance between electrode barrels. This enabled membrane potential to be accurately $(<10 \%$ over-estimate) measured with one barrel when current was passed through the other barrel. Coupling resistance between pairs of ordinary electrodes was insignificant.

\section{RESULTS}

\section{Comparison of hyperpolarizing current and light}

Response amplitude and kinetics. The question addressed here is whether the changes in response amplitude and kinetics associated with the remote adapting effects of background light can be mimicked by hyperpolarizing a cone with extrinsic current applied through the recording electrode. Since the hyperpolarization produced by extrinsic current is greatest in the impaled cone and its closest neighbors, responses to small test flashes (15 $\mu \mathrm{m}$ dia spot) centered on the impaled cone were used for study.

Figure 1 shows larger, relatively noise-free averaged responses from one cone to illustrate the effects of current. Similar effects of current on smaller amplitude responses from two other cells are shown in Figs 2 and 3. Figure 1(A) shows the control response in the dark to a $20 \mathrm{msec}, 15 \mu \mathrm{m}$ dia spot flashed in the center of the cone's receptive field (thin curve), and illustrates the effect of passing $0.2 \mathrm{nA}$ of steady hyperpolarizing current (thick curve). The size of the response with current was reduced by $15.1 \%$ and its time to peak was reduce । by $11 \%$ from 121 to $107 \mathrm{msec}$. Both these changes are large compared to the variability within responses obtained under the same conditions. In Fig. I(B) the response with current shown in Fig. 1(A) has been scaled by 1.4 to illustrate that the falling phases of the two responses have a similar time-course.

Figure 2(A) directly compares, for a different cone, the effects of steady light and hyperpolarizing current on the kinetics of the response. Again, the flash stimulus was a $15 \mu \mathrm{m}$ dia spot centered on the impaled cone. Responses were obtained in the presence of $0.1 \mathrm{nA}$ of hyperpolarizing current (thick curve), and with a superimposed steady slit (dashed curve). The intensity of the background slit was adjusted until it reduced the amplitude the same amount as the current did. The thin line shows the control response obtained with no background or current.

To compare the tails of the responses, the light adapted response has been scaled by a factor of 1.7 and plotted with the control in Fig. 2(B). Even though the scaled response is larger than control it returns to baseline more quickly. This speeding up of the response is a well-documented property of light-adapted responses in turtle (Baylor \& Hodgkin, 1974). The response with current has been scaled by a factor of 1.3

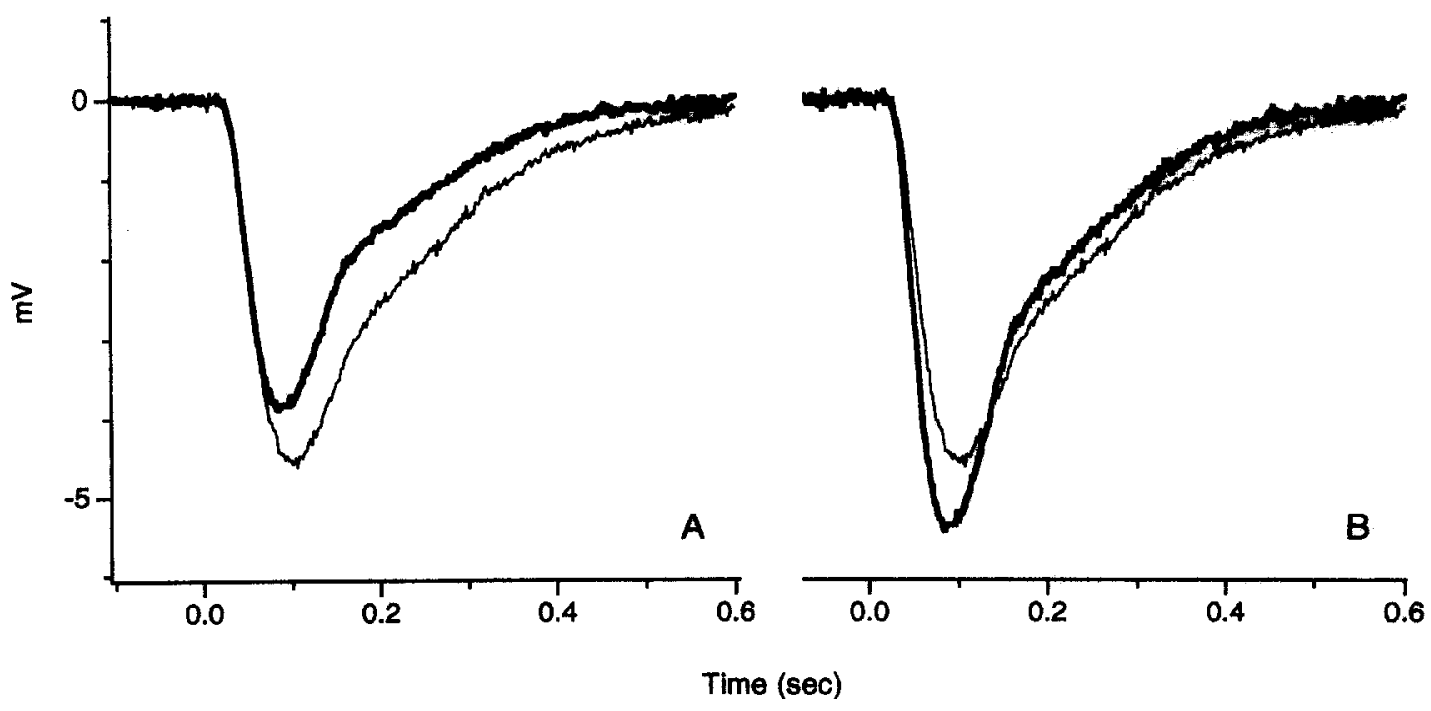

FIGURE 1. Steady hyperpolarizing current $(0.2 \mathrm{nA})$ decreases the amplitude of the response to light in a red cone. (A) The thin line is the control response (average of 2) to a full intensity $15 \mu \mathrm{m}$ dia spot and the thick line is the response (average of 3 ) when current is passed through the cell. (B) The same responses but in this case the current (thick line) has been scaled by a factor of 1.4 to allow a comparison of the return to baseline. 


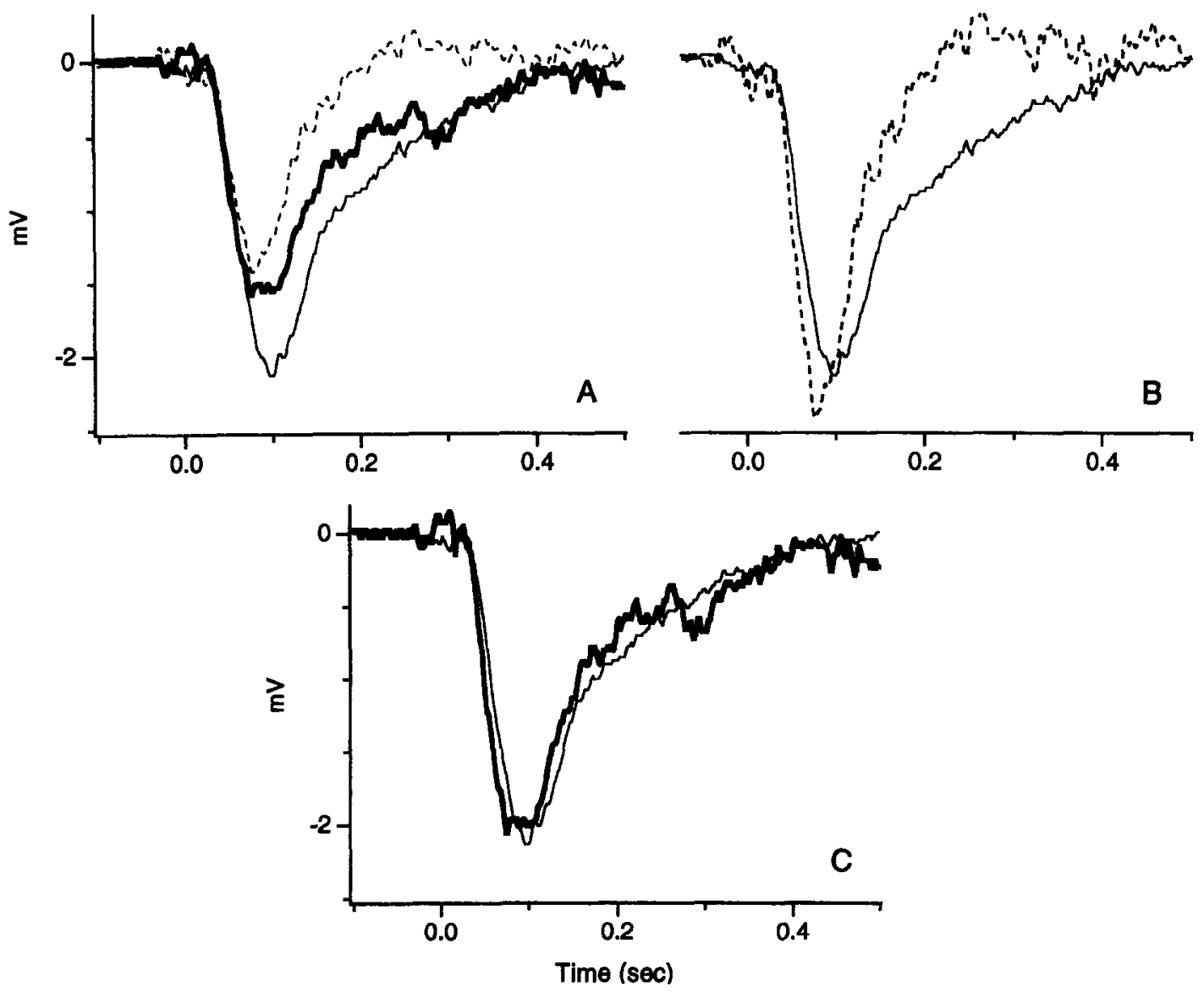

FIGURE 2. Comparison of the desensitizing effects of current and light on a red-sensitive cone. (A) The thin line is the control response (average of 4 ) to a small spot $(15 \mu \mathrm{m}$ dia), the thick line is the response (average of 5$)$ in the presence of hyperpolarizing current $(0.2 \mathrm{nA})$ and the dashed line is the response (average of 3 ) in the presence of a dim adapting slit that hyperpolarized the cone by approx. $1 \mathrm{mV}$. (B) The control response (thin line) compared with the light-adapted response (dashed line) scaled

by a factor of 1.7 (C) The control (thin line) compared with the hyperpolarized response (thick line) scaled by 1.3.

and plotted with the control in Fig. 2(C). In this case the recovery portions of these two curves are very similar.

Results comparable to those illustrated in Figs 1 and 2 were obtained in $25 \%$ (11 of 44 ) of the cones studied. (See below for a discussion of the variability of the results.) In these cones extrinsic hyperpolarizing current reduced the amplitude and time to peak of the response, but unlike background light it did not affect the time-course of the falling phase of the response.

$V-\log I$ curves. In cones where extrinsic current reduced the amplitude of the flash response, extrinsic current and background light had different effects on the intensity-response function. Figure 3(A) shows a set of responses to flashes of varying intensity in the presence and absence of a $0.1 \mathrm{nA}$ hyperpolarizing current. Figure 3(B) plots intensity-response functions obtained from these records.

No saturating responses are present in the intensity-response series shown in Fig. 3, because as the intensity of a small spot is increased, light scatters to neighboring cones. The coupled network allows the increasingly greater scattered light responses from neighbors to contribute to the measured responses (Pluvinage \& Green, 1990).

The effect of current is to scale the responses by a factor of 0.9 , more or less independently of stimulus intensity. Figure 3(C) shows the intensity-response curves for the same cell obtained in the presence and absence of a weak background. The background was selected to reduce the amplitude of the larger responses by a factor of about 0.9 . In contrast to the effects of current, the background light tends to shift the intensity-response curve by $0.4 \log$ units along the log intensity axis.

\section{Dependency on hyperpolarization}

Using single-barreled electrodes it is difficult to infer the change in membrane potential produced by extrinsic currents. To address this problem several cones in the isolated retina of the red-eared turtle were penetrated singly with a theta glass electrode, or in pairs with two ordinary electrodes. In the former case one barrel of the theta electrode was used for passing current while the other barrel recorded the membrane potential. In the latter case electrical coupling between cones sometimes permitted one cone to be hyperpolarized with current injected into a neighbor.

In the theta electrode recording illustrated in Fig. 4(A), $0.1 \mathrm{nA}$ of current produced a steady hyperpolarization of about $12 \mathrm{mV}$ and reduced the response 
amplitude by almost $30 \%$. Figure 4(B) shows a result for a cell that was hyperpolarized $5 \mathrm{mV}$ by injecting $0.4 \mathrm{nA}$ into a neighboring cell. This reduced the response by $11 \%$.

\section{Variability of effect}

The effect of hyperpolarizing current on the response to a $15 \mu \mathrm{m}$ dia spot was measured on a total of 44 mred-sensitive cones in the eyecup. In only 11 of the cells tested with hyperpolarizing current was the responses unequivocally reduced. Of these, in the nine cells injected with $0.1 \mathrm{nA}$ of current the response amplitude was reduced between 5.2 and $27.3 \%$ from control, with an average reduction of $14.6 \%$. The average time-to-peak of $137 \mathrm{msec}$ for these cells was reduced by $6.5 \%$. In 11 of the cones the response was increased by hyperpolarizing current. The increases in amplitude were between 3 and $41 \%$ of control. A current of $0.1 \mathrm{nA}$ produced an average increase in amplitude of $21.1 \%$. In 22 cones. hyperpolarizing current had no effect whatsoever on the response.

We saw no correlation between flash sensitivity and the effects of hyperpolarizing current. Thus we do not think that the variability observed in the effects of current are somehow related to the health of the cell or to the quality of the electrode penetration.

\section{DISCUSSION}

The experiments described here were undertaken to test whether the adaptational effects of a background light could be mimicked simply by hyperpolarizing a cone with extrinsic current. Although we find that the effects of hyperpolarizing currents are quite variable. there is no indication that hyperpolarization per se can adapt the voltage response in the same way that light does.
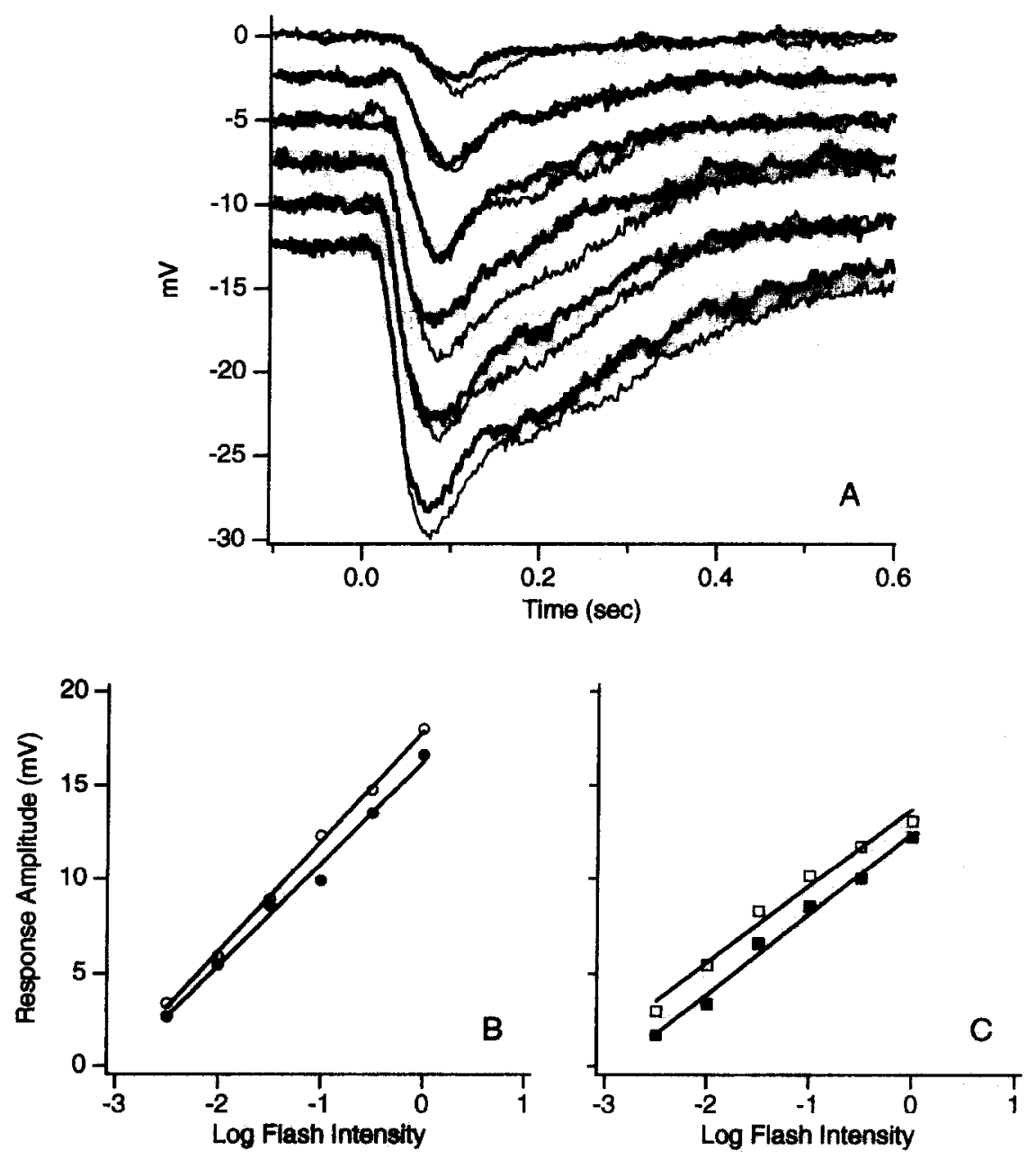

FIGURE 3. Comparison of intensity-response curves with 0.1 nA hyperpolarizing current and with dim backgrounds. (A) The set of responses to a $15 \mu \mathrm{m}$ spot at various intensities. Each pair shows the control response (thin line) and the response when current is passed through the impaling microelectrode (thick line). The stimulus intensity for the top pair was log $l=-2.5$. For each subsequent pair the intensity was increased by approx. $0.5 \log$ units and the trace was displaced downward by $2.5 \mathrm{mV}$. The traces are single responses except for the upper pairs which are the average of three. Points in (B) were obtainod from the responses in (A) and show the response amplitude in the presence (solid circles) and absence (open circles) of hyperpolarizing current. (C) The intensity-response curve obtained with a dim $15 \mu \mathrm{m}$ spot background light (solid squares): the open squares are the control. The lines are fit by regression. 


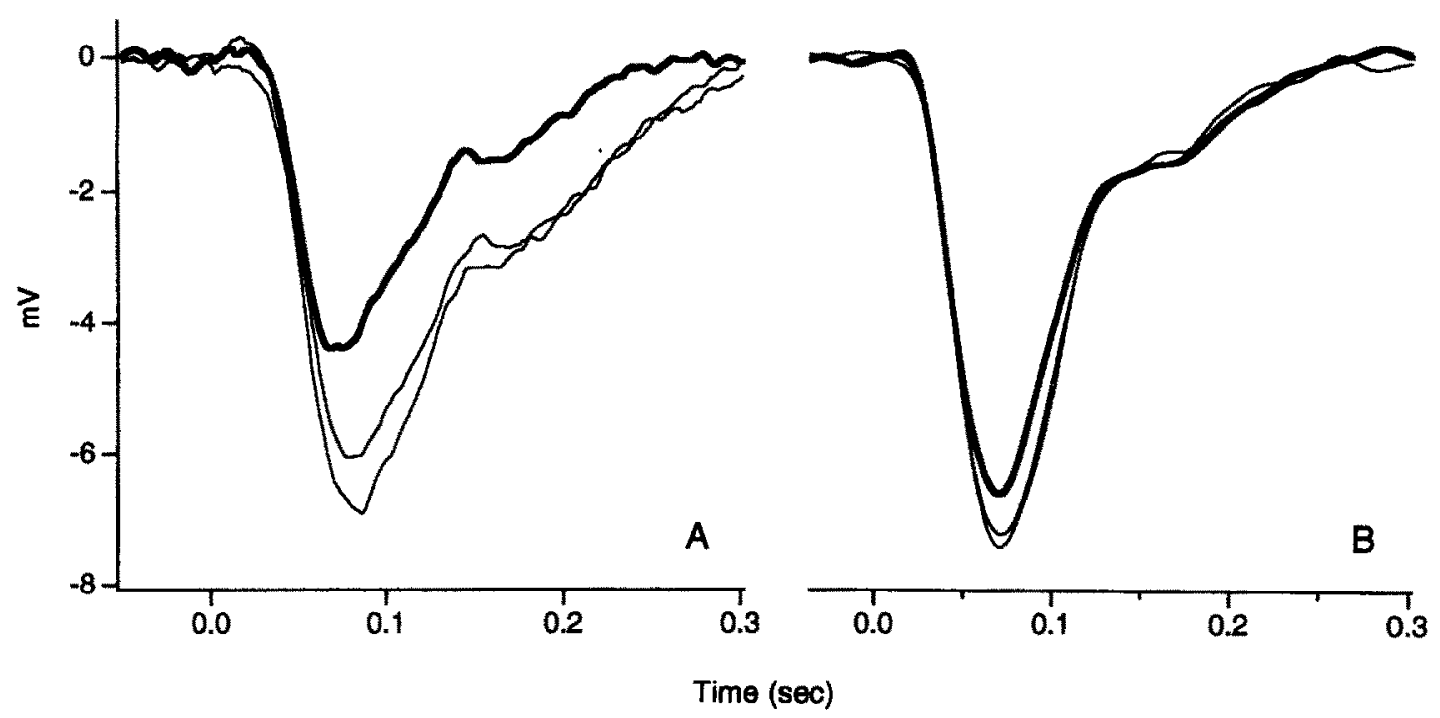

FIGURE 4. The effect of extrinsic current on membrane potential and flash response in cones from the red-eared turtle. (A) The responses to a $151 \mu \mathrm{m}$ dia spot ( $20 \mathrm{msec}$ fash) recorded with a theta electrode. Current was passed through one barrel while voltage was measured with the other. The thick curve shows the response was reduced by almost $30 \%$ when the cell was hyperpolarized by $12 \mathrm{mV}$ with $0.1 \mathrm{nA}$ of current. The thin curves show control responses before and after the injection of current. The response afterward was slightly smaller than the response before. (B) The responses to a $151 \mu \mathrm{m}$ dia spot ( $20 \mathrm{msec}$ flash) recorded from one cone of a pair impaled simultaneously with two electrodes. The thick curve shows the response was reduced by $11 \%$ when the cell was hyperpolarized by $5 \mathrm{mV}$ with $0.4 \mathrm{nA}$ of current injected into the coupled neighbor. The thin curves show control responses before and after the injection of current.

\section{Voltage-sensitive mechanisms}

In $25 \%$ of the cones studied, $0.1 \mathrm{nA}$ extrinsic hyperpolarizing current reduced the amplitude and time-topeak of the response, but otherwise had little effect on the kinetics. This result is consistent with the findings that hyperpolarization activates an inner segment conductance that shunts the photocurrent (Schneeweis, 1991). Such inner segment voltage-sensitive conductances have been found and studied in several photoreceptors including salamander rods and cones (Attwell \& Wilson, 1980; Attwell, Werblin \& Wilson, 1982; Baylor, Matthews \& Nunn, 1984; Hestrin, 1987; Barnes \& Hille, 1989), lizard cones (Maricq \& Korenbrot, 1990), toad rods (Fain, Quandt, Bastian \& Gerschenfeld, 1978; Torre \& Owen, 1983), and snapping turtle rods (Detwiler, Hodgkin \& McNaughton, 1980; Copenhagen \& Owen, 1980). While less-well studied, a role for a voltage-sensitive conductance in cones of red-eared turtle has been proposed by Baylor and Hodgkin (1974), and additional evidence for this can be seen in the current-step responses in Detwiler and Hodgkin (1979). We have confirmed these results in preliminary experiments on cones from red-eared turtle studied in the isolated retina preparation (Schneeweis, 1991).

If the cone photocurrent amplitude and kinetics are also voltage-dependent, changes in the cone voltage response are not dictated solely by the behavior of inner segment voltage-sensitive conductances. On the basis of experiments in salamander cones (Attwell et al., 1982) and the current-voltage relationship of the light sensitive channels of catish cones (Haynes \& Yau, 1985), the photocurrent flash response would be expected to increase with hyperpolarization. This tendency alone would lead to an increase in the voltage response. The ultimatc effect on the voltage response would depend on a combination of changes in the photocurrent and inner segment conductance, and could conceivably result in either an increase or a decrease of the response. Since in this study hyperpolarizing current increased, decreased or, in many cases, had no effect at all on the flash response it may be that these voltage-dependent mechanisms are fairly delicately balanced in snapping turtle cones.

\section{Insufficient hyperpolarization}

In the eyecup experiments it was not possible to measure the change in resting potential produced by extrinsic current, presenting the possibility that the cones were not hyperpolarized to a degree sufficient to activate some adaptation mechanism. This seems unlikely since a background light that hyperpolarizes by only $2-3 \mathrm{mV}$ reduces the flash sensitivity of turtle cones by about $\mathrm{e}^{-1}$ (Baylor \& Hodgkin, 1974; Copenhagen \& Green, 1987; Itzhaki \& Perlman, 1987). Assuming a cone input resistance of about $25 \mathrm{M} \Omega$ (Lamb \& Simon, 1977; Detwiler \& Hodgkin, 1979), $0.1 \mathrm{nA}$ would be expected to hyperpolarize a cone in the eyecup by about $2.5 \mathrm{mV}$. Futhermore, in experiments where cones in the isolated retina of the red-eared turtle were penetrated with theta glass electrodes, $0.1 \mathrm{nA}$ of current hyperpolarized the cones by 5-15 mV (Schneeweis, 1991).

\section{Spread of adaptation}

This study has shown that hyperpolarization per se may reduce response amplitude in some cones, probably through a voltage-sensitive increase in the inner segment conductance. Since photons caught in one cone will 
produce a hyperpolarization in a coupled neighboring cone, this effect may be responsible for some small part of remote adaptation in some cones. The question remains, however, what is it, if not hyperpolarization, that has greater control over sensitivity and mediates the apparent spread of adaptation between cones?

Synaptic messengers. It is possible that remote adaptation is mediated by synaptic transmission, either via synapses between cones or by feedback through horizontal cells. There is ample anatomical evidence for putative chemical synapses between photoreceptors in turtle (Mariani \& Lasansky, 1984; Kolb \& Jones, 1985), while evidence for a physiological role is lacking. A role for horizontal cells seems less likely since the slits and spots used to study remote adaptation (Copenhagen \& Green, 1987) stimulate horizontal cells only minimally (Leeper \& Copenhagen, 1979).

Diffusible messenger. A second possibility is that remote adaptation is mediated by some diffusible messenger, recent examples of which are nitric oxide and carbon monoxide. Such a messenger might diffuse from one cone to other cones, or from bipolar cells to cones. While nitric oxide synthase has been found in retinal neurons of several species [amacrine cells in several mammals (Sandell, 1985); horizontal cells in several lower vertebrates (Miyachi, Miyakama \& Murakami, 1991); bovine rod outer segments (Goureau, Lepoivre, Mascarelli \& Courtois, 1992; Venturini, Knowles, Palmer \& Moncada, 1991)], it remains to be determined whether a mechanism exists whereby nitric oxide might modulate cone light responses. Recently it has been shown that photoreceptors may contain the nitric oxide-sensitive soluble form of guanylate cyclase, albeit at much lower levels than the particulate form of the protein (Margulis, Sharma \& Sitaramayya, 1992; Ahmad \& Barnstable, 1993). This raises the possibility that nitric oxide could affect the light response by modulating guanylate cyclase activity. Experiments looking at the pharmacology of remote adaptation are required to test this possibility.

Scattered light. Scattered light must certainly play some role. Estimates of light scattering come from a number of different experiments. Recordings from uncoupled cones (Baylor \& Hodgkin, 1973; Pluvinage \& Green, 1990), the limited extent of lateral spread of adaptation in rods (Copenhagen \& Green, 1985), and a comparison of the cone spatial sensitivity profile with the fall of electrical coupling between cone pairs (Detwiler \& Hodgkin, 1979) all indicate that light scatter from a narrow slit is down by $\mathrm{e}^{-1}$ at a distance of about $10 \mu \mathrm{m}$. On the other hand, the space constant for the spatial sensitivity profile for adaptation and excitation in red-sensitive cones is about $25 \mu \mathrm{m}$ (Copenhagen \& Green, 1987). Taken together these two findings imply that light scatter can only account for a small component of the spread of adaptation and most of the desensitizing effect obtained with a large adapting stimulus is due to the influence of neighboring cones.

\section{REFERENCES}

Ahmad, I. \& Barnstable, C. J. (1993). Differential laminar expression of particulate and soluble guanylate cyclase genes in rat retina. Experimental Eye Research, 56, 51-62.

Attwell, D. \& Wilson, M. (1980). Behavior of the rod network in the tiger salamander retina mediated by membrane properties of individual rods. Journal of Physiology, 309, 287-315.

Attwell, D., Werblin, F. S. \& Wilson, M. (1982). The properties of single concs isolated from the tiger salamander retina. Journal of Physiology, 328, 259-283.

Barnes, S. \& Hille, B. (1989). Ionic channels of the inner segment of tiger salamander cone photoreceptors. Journal of General Physiology, 94, 719-743.

Baylor, D. A., Fuortes, M. G. F. \& O'Bryan, P. M. (1971). Receptive fields of cones in the retina of the turtle. Journal of Physiology, 2/4, 265-294.

Baylor, D. A. \& Hodgkin, A. L. (1973). Detection and resolution of visual stimuli by turtle photoreceptors. Journal of Physiology, 234, $163-198$.

Baylor, D. A. \& Hodgkin, A. L. (1974). Changes in time scale and sensitivity in turtle photoreceptors. Journal of Physiology, 242, 729-758

Baylor, D. A., Matthews, G. \& Nunn, B. J. (1984). Location and function of voltage-sensitive conductances in retinal rods of the salamander, Ambystoma tigrinum. Journal of Physiology, 354, 203-223.

Colburn, T. R. \& Schwartz, E. A. (1972). Linear voltage control of current passed through a micropipette with variable resistance. IEEE Biomedical Transactions, 10, 504-509.

Copenhagen, D. R. \& Green, D. G. (1985). The absence of spread of adaptation between rod photoreceptors in turtle retina. Journal of Physiology, 369, 161-181.

Copenhagen, D. R. \& Green, D. G. (1987). Spatial spread of adaptation within the cone network of turtle retina. Journal of Physiology, 393, 763776.

Copenhagen, D. R. \& Owen, W. G. (1980). Current-voltage relations in the rod photoreceptor network of the turtle retina. Journal of Physiology, 308, 159-184.

Detwiler, P. B. \& Hodgkin, A. L. (1979). Electrical coupling between cones in turtle retina. Journal of Physiology, 291, 7-100.

Detwiler, P. B., Hodgkin, A. L. \& McNaughton, P. A. (1980). Temporal and spatial characteristics of the voltage response of rods in the retina of the snapping turtle. Journal of Physiology, 300, 213-350.

Fain, G. L., Quandt, F. M., Bastian, B. L. \& Gerschenfeld, H. M. (1978). Contribution of a caesium-sensitive conductance increase to the rod photoresponse. Nature, 272, 467-469.

Goureau, O., Lepoivre, M., Mascarelli, F. \& Courtois, Y. (1992). Nitric oxide synthase activity in bovine retina. Structures and Functions of Retinal Proteins, 221, 395-398.

Haynes, L. \& Yau, K.W. (1985). Cyclic GMP-sensitive conductance in outer segment membrane of catfish cones. Nature, 317, 61-64.

Hestrin, S. (1987). The properties and function of inward rectification in rod photoreceptors of the tiger salamander. Journal of Physiologv. 390, 319-333.

Itzhaki, A. \& Perlman, I. (1987). Light adaptation of red cones and L1-horizontal cells in the turtle retina: Effect of the background spatial pattern. Vision Research, 27, 685 696

Kolb, H. \& Jones, J. (1985). Electron microscopy of golgi-impregnated photoreceptors reveals connections between red and green cones in the turtle retina. Journal of Neurophysiology, 54, $304-317$.

Lamb, T. D. \& Simon, E. J. (1977). Analysis of electrical noise in turtle cones. Journal of Physiology, 272, 435-468.

Leeper, H. F. \& Copenhagen, D. R. (1979). Mixed rod-cone responses in horizontal cells of snapping turtle retina. Vision Research, 19, $407-412$.

Margulis, A., Sharma, R. K. \& Sitaramayya, A. (1992). Nitroprassidesensitive and insensitive guanylate cyclases in retinal rod outer segments. Biochemical and Biophysical Research Communications, $185,909.914$

Mariani, A. \& Lasansky, A. (1984). Chemical synapses between turtle photoreceptors. Brain Research, 310, 351-354. 
Maricq, A. V. \& Korenbrot, J. I. (1990). Potassium currents in the inner segment of single retinal cone photoreceptors. Journal of Neurophysiology, 64, 1929-1940.

Miyachi, E., Miyakawa, A. \& Murakami, M. (1991). Modulation of electrical coupling between retinal horizontal cells by intracellular messengers. Neuroscience Research (Suppl.), 15, S41-S49.

Owen, W. G. \& Copenhagen, D. R. (1977). Characteristics of the electrical coupling between rods in the turtle retina. In Barlow, H. B \& Fatt, P. (Eds), Vertebrate photoreception. New York: Academic Press.

Pluvinage, V. \& Green, D. G. (1990). Square root intensity coding in turtle cones: Physiological mechanisms. Vision Research, 30, 683-691.

Sandell, J. H. (1985). NADPH diaphorase cells in the mammalian inner retina. Journal of Comparative Neurology, 238, 466-472.
Schneeweis, D. (1991). Coupling in photoreceptors: Spectral and adaptational properties. Ph.D. thesis, University of Michigan, Ann Arbor, Mich

Torre, V. \& Owen, W. G. (1983). High-pass filtering of small signals by the rod network in the retina of the toad, Bufo marinus. Biophysical Journal, 41, 305-324.

Venturini, C. M., Knowles, R. G., Palmer, R. M. \& Moncada, S. (1991). Synthesis of nitric oxide in the bovine retina. Biochemical and Biophysical Research Communications, 180, 920-925.

Acknowledgements - We thank John McReynolds for reading and commenting on an earlier version of this manuscript. This study was suppported in part by NIII research grant EY00379. 